EPJ Web of Conferences 59, 15004 (2013)

DOI: $10.1051 /$ epjconf/20135915004

(C) Owned by the authors, published by EDP Sciences, 2013

\title{
High Mach-number collisionless shock driven by a laser with an external magnetic field
}

\author{
T. Morita1, a , Y. Sakawa ${ }^{1}$, Y. Kuramitsu ${ }^{1}$, T. Ide ${ }^{2}$, K. Nishio $^{3}$, M. Kuwada ${ }^{3}$, \\ H. Ide ${ }^{3}$, K. Tsubouchi ${ }^{2}$, H. Yoneda ${ }^{4}$, A. Nishida ${ }^{2}$, T. Namiki ${ }^{4}$, T. Norimatsu ${ }^{1}$, \\ K. Tomita ${ }^{5}$, K. Nakayama ${ }^{5}$, K. Inoue ${ }^{5}$, K. Uchino ${ }^{5}$, M. Nakatsutsumi ${ }^{6}$, \\ A. Pelka ${ }^{6}$, M. Koenig ${ }^{6}$, Q. Dong ${ }^{7}$, D. Yuan ${ }^{7}$, G. Gregori ${ }^{8}$ and H. Takabe ${ }^{1}$ \\ 1 Institute of Laser Engineering, Osaka University, Japan \\ 2 Graduate School of Engineering, Osaka University, Japan \\ 3 Graduate School of Science, Osaka University, Japan \\ 4 Institute for Laser Science, University of Electro-communications, Japan \\ 5 Interdisciplinary Graduate School of Engineering Sciences Kyushu University, Japan \\ ${ }^{6}$ LULI, École Polytechnique, CNRS, CEA, UPMC, France \\ 7 Institute of Physics, Chinese Academy of Sciences, China \\ ${ }^{8}$ Department of Physics, University of Oxford, UK
}

\begin{abstract}
Collisionless shocks are produced in counter-streaming plasmas with an external magnetic field. The shocks are generated due to an electrostatic field generated in counter-streaming laser-irradiated plasmas, as reported previously in a series of experiments without an external magnetic field [T. Morita et al., Phys. Plasmas, 17, 122702 (2010), Kuramitsu et al., Phys. Rev. Lett., 106, 175002 (2011)] via laserirradiation of a double-CH-foil target. A magnetic field is applied to the region between two foils by putting an electro-magnet $(\sim 10 \mathrm{~T})$ perpendicular to the direction of plasma expansion. The generated shocks show different characteristics later in time $(t>20 \mathrm{~ns})$.
\end{abstract}

\section{INTRODUCTION}

The aim of the research is to study collisionless shocks as observed in supernova remnants (SNRs) and to find the physics of collisionless shock formation. This also aims at the physics of particle acceleration relating to the origin of cosmic-rays. In the universe, collisionless shocks are, for example, formed due to supernova explosions [1]. The diffusive shock acceleration (DSA) is the standard model for the acceleration of cosmic-rays. It is, however, impossible to directly measure the accelerated particles by shocks at SNRs and to measure various parameters such as electric and magnetic fields at the shocks.

Laboratory experiments are one of the alternative ways to investigate the astrophysical phenomena [2]. We have studied collisionless shocks with high-power laser-produced counter-streaming plasmas $[3,4]$. A large density jump due to an electrostatic (ES) shock has been observed by the optical diagnostics such as shadowgraphy (SG) and interferometry (IF) [3]. Using streaked optical pyrometry (SOP) and streaked interferometry (SIF), time evolution of high Mach-number ES shocks has been observed [4]. These experiments, however, have been performed without an external magnetic field.

\footnotetext{
ae-mail: moritat@ile.osaka-u.ac.jp
}

This is an Open Access article distributed under the terms of the Creative Commons Attribution License 2.0, which permits unrestricted use, distribution, and reproduction in any medium, provided the original work is properly cited. 
(a)

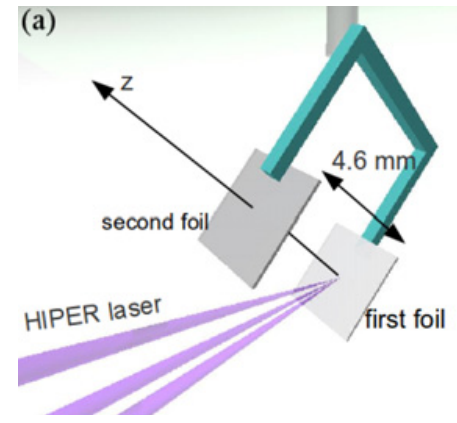

(b)

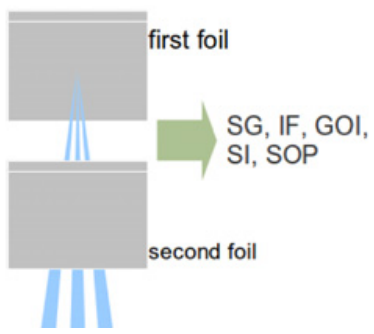

Figure 1. (a) The schematic view of the target. The first foil is irradiated by three beams to produce counterstreaming plasmas between two foils. (b) The top view of the target. The plasmas are diagnosed by the SG, GOI, SI, and SOP from the direction perpendicular to the plasma expansion.

It is important to investigate the effect of external magnetic fields on a shock formation because there are weak ambient magnetic fields in the galaxy. Collisionless plasma interactions have been investigated to simulate SNR shocks $[5,6]$, however, no shocks were observed early in time $t=500 \mathrm{ps}$ in which the plasma beta in the experiment, the ratio of the ram pressure of a plasma flow to the magnetic pressure $\left(\beta=\left(n_{\mathrm{i}} m_{\mathrm{i}} v_{\mathrm{i}}^{2} / 2\right) /\left(B^{2} / 2 \mu_{0}\right)\right)$, was comparable to that of typical SNRs. Harilal et al. have investigated the dynamics of expanding laser-produced plasmas across a transverse magnetic field in a high $\beta$ condition [7]. The plasmas was clearly slowed down, according to the time-of-flight measurements performed at late time.

In this report, we show the collisionless shock generation with an external magnetic field in which the plasma beta is relatively high $(6<\beta<200)$ at $10 \mathrm{~ns}<t<60 \mathrm{~ns}$ assuming the electron density $n_{\mathrm{e}} \sim 10^{18} \mathrm{~cm}^{-3}$.

\section{EXPERIMENTAL SETUP}

The experiment was performed at institute of laser engineering, Osaka University, with Gekko-XII HIPER laser system, frequency tripled Nd : Glass laser $(351 \mathrm{~nm})$ which has the energy of $\sim 120 \mathrm{~J} /$ beam in $500 \mathrm{ps}$ with the focal spot of $300 \mu \mathrm{m}$ in diameter and the intensity of $10^{15} \mathrm{~W} / \mathrm{cm}^{2}$ to produce highvelocity counter-streaming plasmas. Three laser beams are focused on one of the inner surface of a double-CH-foil target (first-foil) as shown in Fig. 1(a) to produce a high-velocity plasma flow. The second plasma flow from the other foil (second-foil) is produced by the radiation and a plasma flow from the first plasma generated at the first-foil, resulting in the generation of counter-streaming plasmas between two foils. The both foils are made of $\mathrm{CH}$ with the thickness of $200 \mu \mathrm{m}$ and are separated by $4.6 \mathrm{~mm}$. The axes $z$ and $r$ are defined as the distance from the first foil and that from the axis $z$, respectively, as shown in Fig. 1(a).

The plasmas are diagnosed by a SOP and self-emission gated-optical-imager (GOI) by observing the emission, and two SGs using a probe laser: frequency doubled Nd: YAG laser $(532 \mathrm{~nm})$ with the pulse duration of $\sim 10 \mathrm{~ns}$. The SOP is a streaked data of an one-dimensional image between the two foils at a certain wavelength using a band-pass filter which has a central wavelength of $450 \mathrm{~nm}$. The GOI is a twodimensional image of the emission in the same range of the wavelength as the SOP using an intensified charge coupled devices (ICCDs) camera with an exposure time of $\sim 1.6 \mathrm{~ns}$. Two ICCD cameras with minimum exposure times of $\sim 200 \mathrm{ps}$ are used for the SGs. These detectors measure from the transverse direction as shown in the top view of the target in Fig. 1(b). Combining the plasma parameters such as $n_{\mathrm{e}}$, plasma size, and the emission intensity obtained from the SOP and GOI, the plasma temperature can be estimated [8]. 

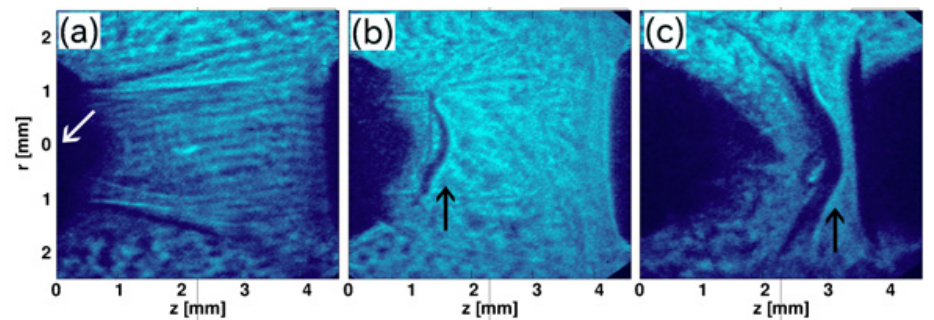

Figure 2. The SG data taken at (a) $10 \mathrm{~ns}$, (b) $20 \mathrm{~ns}$, and (c) $60 \mathrm{~ns}$ without an external magnetic field. The white arrow shows the focal spot of the incident laser, and the black arrows show the shocks.
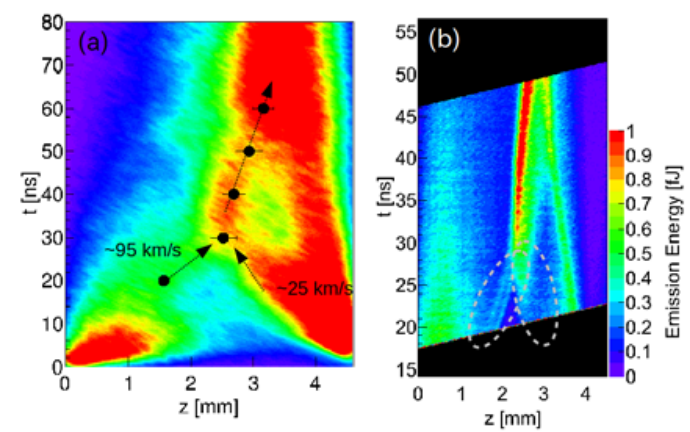

Figure 3. (a) The SOP data taken in this experiment and (b) in a previous experiment without an external magnetic field. The closed circles show the shock observed by the SG. The SOP data (b) was taken in a same target but using four laser beams. Two shocks were observed as shown with ellipses.

An electro-magnet with an effective diameter of $\sim 2 \mathrm{~mm}$ is placed at $\sim 5 \mathrm{~mm}$ from the edge of the target. An external magnetic field of $\sim 10 \mathrm{~T}$ perpendicular to the target normal direction is produced by a pulsed-power generator. The duration of the applied magnetic field is $\sim 0.5 \mu \mathrm{s}$, which is large enough for the time evolution of plasmas and the time range of all the diagnostics.

\section{RESULT}

In order to investigate the shock formation and propagation, the SG data are taken at different times using identical targets and laser energies. Figures 2(a)-2(c) show the results of the SG at $t=10 \mathrm{~ns}$, $20 \mathrm{~ns}$, and $60 \mathrm{~ns}$, respectively. The white arrow in Fig. 2(a) represents the position of the incident laser. The shock positions are shown with the black arrows in the SG data. There are fine structures at $r=1-$ $2 \mathrm{~mm}$ at $t=10 \mathrm{~ns}$, however, no shock is observed. At $t=20 \mathrm{~ns}$, a sharp density jump due to a shock is observed, and it propagates from left to right as shown in Figs. 2(b) and 2(c).

Figure 3(a) shows the SOP data. In the figure, the closed circles represent the shock positions $z$ at time $t$ observed in the SG data shown in Fig. 2 (the SG data at $t=30 \mathrm{~ns}, 40 \mathrm{~ns}$, and $50 \mathrm{~ns}$ are not shown). The SOP data is not clear because of the misalignment of the detector, however, the bright regions well correspond with the shock positions as shown with the closed circles. Figure 3(b) shows the SOP data taken in a previous experiment with an identical target and different laser energy using four laser beams (total laser energy is $572 \mathrm{~J}$ ). At $t=25-35 \mathrm{~ns}$, clear shocks are observed and interact each other at $t>35 \mathrm{~ns}$.

Figures 4(a)-4(d) show the SG data with an external magnetic field at $t=20 \mathrm{~ns}, 30 \mathrm{~ns}, 50 \mathrm{~ns}$, and $60 \mathrm{~ns}$, respectively. Shocks are clearly observed in the SG data as well as those without the magnetic field in Figs. 2(a)-2(c). Shock positions as a function of $t$ are plotted in Fig. 3(e) with (open circles) and 


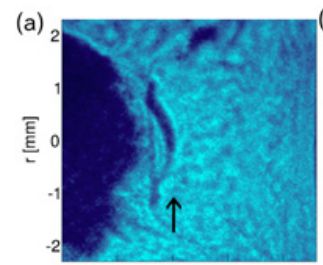

(c)
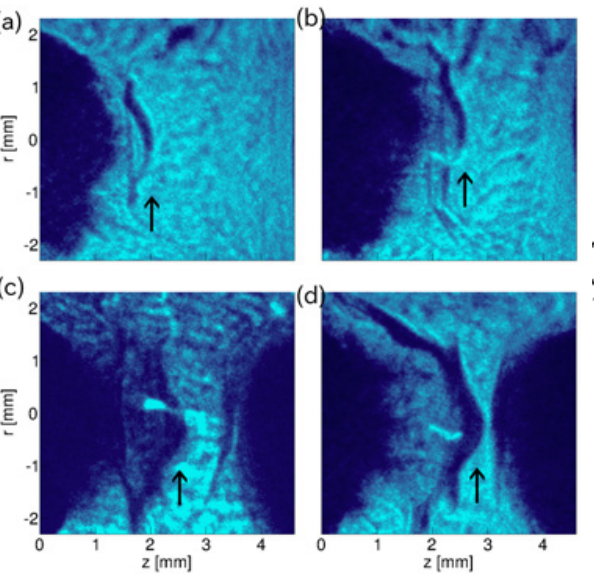

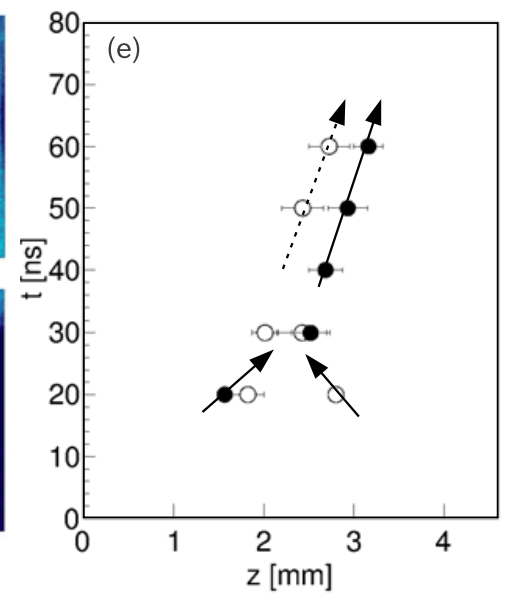

Figure 4. The SG data taken at (a) $t=20 \mathrm{~ns}$, (b) $30 \mathrm{~ns}$, (c) $50 \mathrm{~ns}$, and (d) $60 \mathrm{~ns}$ with the external magnetic field. (e) The time evolution of the shocks observed by the SGs with (open circles, dashed line) and without (closed circles, solid line) the external magnetic field.

without (closed circle) the magnetic field. Solid lines and dashed lines are guides for eyes. The shock positions are different with and without the magnetic field at $t>20 \mathrm{~ns}$.

The parameter $\beta$ is estimated by assuming the average ion mass $m_{\mathrm{i}}=A m_{\mathrm{p}}$ where $A=7.5$ and $m_{\mathrm{p}}$ are the average mass number and the proton mass, respectively, the average degree of charge $Z=3.5$, and the ion velocity defined as $v_{\mathrm{i}}=z / t$, as $\beta \simeq(A / Z)\left(\mu_{0} m_{\mathrm{p}} / B^{2}\right) n_{\mathrm{e}}(z / t)^{2}=3.9 \times 10^{3} \times \tilde{n_{\mathrm{e}}}(\tilde{z} / \tilde{t})^{2}$, where $\tilde{n_{\mathrm{e}}}$ is $n_{\mathrm{e}}$ in the unit of $10^{18} \mathrm{~cm}^{-3}, \tilde{z}$ is the distance from the first foil in mm, and $\tilde{t}$ is $t$ in ns. The parameters $\beta$ at different time assuming the electron density $\tilde{n_{\mathrm{e}}}=1$ at the position of $z=2.3 \mathrm{~mm}$ (at the center of two foils) are $\beta=210$ at $t=10 \mathrm{~ns}, \beta=52$ at $t=20 \mathrm{~ns}$, and $\beta=5.7$ at $t=60 \mathrm{~ns}$. The results with and without the external magnetic field show the clear difference later in time ( $t>20 \mathrm{~ns})$ at $\beta<50$ especially with respect to the shock positions. Further analyses and measurements are required to determine the plasma parameters.

The collisionality of two counter-streaming flows is estimated with the ion-ion mean-free-path (MFP) $\lambda_{\mathrm{ii}}$ using the Spitzer's formula [9] as $\lambda_{\mathrm{ii}}=2 \pi \epsilon_{0}^{2} m_{\mathrm{i}}^{2} u_{\mathrm{i}}^{4} /\left(n_{\mathrm{i}} Z^{4} e^{4} \ln \Lambda\right)$, where $u_{\mathrm{i}}$ is the relative velocity of counter flows and $\ln \Lambda$ is the Coulomb logarithm. Assuming $n_{\mathrm{e}}=10^{18} \mathrm{~cm}^{-3}$ and the electron temperature $T_{\mathrm{e}}=10 \mathrm{eV}, \lambda_{\mathrm{ii}}=19 \mathrm{~mm}$ at $t=10 \mathrm{~ns}$ and $\lambda_{\mathrm{ii}}=310 \mu \mathrm{m}$ at $t=30 \mathrm{~ns}$. Later in time, the MFP becomes small and comparable to the observed shock width $(\sim 100 \mu \mathrm{m})$ in the SG data; the Coulomb collision is dominant for the shock formation later in time. On the other hand, collisionless shocks in which the MFP is much larger than the shock width are clearly observed at $t<30 \mathrm{~ns}$.

\section{CONCLUSION}

We have produced collisionless ES shocks in counter-streaming laser-produced plasmas in an external magnetic field at $t<30 \mathrm{~ns}$. The density jumps due to the collisionless shocks are clearly observed in the SG as in Fig. 2. Comparing the results with and without the external magnetic field, the effect of the magnetic field on the shock formation is observed later in time $t>20 \mathrm{~ns}$ with $6<\beta<50$.

The authors acknowledge the dedicated technical support by the staff at the GEKKO-XII facility for the laser operation and target fabrication. This research was performed under the joint-research project of the Institute of 


\section{IFSA 2011}

Laser Engineering, Osaka University, and supported by Grant-in-Aid for Scientific Research (B), MEXT of Japan (No. 21340172), by a grant for the Core-to-Core Program from the Japan Society for the Promotion of Science (JSPS), and by a Grant-in-Aid for JSPS Fellows.

\section{References}

[1] F. A. Aharonian et al, Nature 432, 75 (2004)

[2] B. Remington et al, Reviews of Modern Physics 78, 755 (2006)

[3] T. Morita et al, Physics of Plasmas 17, 122702 (2010)

[4] Y. Kuramitsu et al, Physical Review Letters 106, 175002 (2011)

[5] N. C. Woolsey et al, Physics of Plasmas 8, 2439 (2001)

[6] C. Courtois et al, Physics of plasmas 11, 3386 (2004)

[7] S. S. Harilal et al, Physical Review E 69, 026413 (2004)

[8] T. Morita et al, Astrophysics and Space Science 336, 283 (2010)

[9] L. Spitzer, Physics of Fully Ionized Gases (Interscience, 1962) 